\title{
The role of neighboring infected cattle in bovine leukemia virus transmission risk
}

\author{
Sota KOBAYASHI ${ }^{1) *}$, Toshiyuki TSUTSUI ${ }^{1)}$, Takehisa YAMAMOTO ${ }^{1)}$, Yoko HAYAMA ${ }^{1)}$, Norihiko MUROGA ${ }^{1)}$, \\ Misako KONISHI $^{1)}$, Ken-ichiro KAMEYAMA ${ }^{1)}$ and Kenji MURAKAMI ${ }^{2}$ \\ 1) Viral Diseases and Epidemiology Research Division, National Institute of Animal Health, 3-1-5 Kannondai, Tsukuba, Ibaraki \\ 305-0856, Japan \\ ${ }^{2)}$ Cooperative Department of Veterinary Medicine, Faculty of Agriculture, Iwate University, 3-18-8 Ueda, Morioka, Iwate 020-8550, \\ Japan
}

(Received 5 January 2014/Accepted 19 February 2015/Published online in J-STAGE 5 March 2015)

\begin{abstract}
A cohort study was conducted to evaluate the risk of bovine leukemia virus (BLV) transmission to uninfected cattle by adjacent infected cattle in 6 dairy farms. Animals were initially tested in 2010-2011 using a commercial ELISA kit. Uninfected cattle were repeatedly tested every 4 to 6 months until fall of 2012. The Cox proportional hazard model with frailty showed that uninfected cattle neighboring to infected cattle $(\mathrm{n}=53)$ had a significant higher risk of seroconversion than those without any infected neighbors ( $\mathrm{n}=81)$ (hazard ratio: 12.4, $P=0.001$ ), implying that neighboring infected cattle were a significant risk factor for BLV transmission. This finding provides scientific support for animal health authorities and farmers to segregate infected cattle on farms to prevent spread of BLV.
\end{abstract}

KEY WORDS: bovine leukemia virus, cohort study, on-farm transmission

doi: 10.1292/jvms.15-0007; J. Vet. Med. Sci. 77(7): 861-863, 2015

Enzootic bovine leucosis (EBL) is an oncogenic infectious disease of cattle caused by bovine leukemia virus (BLV), a member of the genus Deltaretrovirus in the family Retroviridae $[2,9]$. Bovine leukemia, a disease complex consisting primarily of EBL as well as sporadic bovine leucosis, is designated as a reportable livestock disease in Japan.

According to a recent nationwide survey, an estimated $40 \%$ of Japanese dairy cattle were infected with BLV [7]. Effective mitigation of this high prevalence requires intensive control measures against risk factors associated with BLV transmission. To date, well-known risk factors for BLV transmission, such as reusing needles for vaccines or the plastic sleeves for rectal palpation, are avoided on Japanese dairy farms [3]. This suggests that the iatrogenic risk of BLV transmission is considered to have decreased.

In addition, segregation of infected animals has often been recommended to reduce the chance of BLV transmission to uninfected cattle. However, little experimental evidence exists to support this intervention. While many see this as an inexpensive, quick way to reduce transmission risk, segregation is not without its impact on farms. This measure affects farmers' routine activities, including milking order, which is primarily determined by parity, pregnancy status, mastitis and other conditions rather than BLV infection. If there is no change in the risk of BLV transmission when mixing infected and uninfected cattle, farmers should be relieved

*Correspondence to: Kobayashi, S., Viral Diseases and Epidemiology Research Division, National Institute of Animal Health, 3-1-5 Kannondai, Tsukuba, Ibaraki 305-0856, Japan.

e-mail: sotaco@affrc.go.jp

(C)2015 The Japanese Society of Veterinary Science

This is an open-access article distributed under the terms of the Creative Commons Attribution Non-Commercial No Derivatives (by-nc-nd) License $<$ http://creativecommons.org/licenses/by-nc-nd/3.0/>. from the burden of segregation. Therefore, we conducted a cohort study to evaluate whether the risk for seroconversion of uninfected cattle is influenced by the infection status of adjacent cattle.

This study was conducted in Japan from 2010 to 2012 at 6 dairy farms with tie-stall housing. These farms, known to be infected with BLV, participated on a voluntary basis after the coordination by prefectural livestock hygiene service centers. All the cattle in this study were Holsteins. On each farm, all cattle were initially tested between June 2010 and August 2011 using a commercial ELISA kit, following the manufacturer's directions (JNC, Tokyo, Japan). Cattle with a positive reaction were defined as infected and the remaining cattle as uninfected. Uninfected cattle were tested in 4-6-month intervals until November 2012 or seroconversion. At each sampling, data collected included the results of the serological testing and allocation records of all cattle in the cattle houses. As the allocations of some cattle in the cattle houses were changed at the farmers' convenience, uninfected cattle were not always kept under the same conditions. Therefore, based on the allocation records, if the infection status of the adjacent cattle changed, the test results obtained before the previous test were used and the following observation was censored. Based on these criteria, uninfected cattle were separated into 2 groups; uninfected cattle without infected neighbors as an unexposed group and those with infected neighbor (s) in the cattle house as an exposed group. In this trial, we defined that 'neighboring' is the situation where an uninfected cow is located just next to the infected cow (s). Therefore, the infected cattle in the opposite lane of the cattle house were not incorporated into this criterion.

All statistical analyses were conducted by $\mathrm{R}$ ver. 3.02 [10]. First, the number of seroconverted cattle in each group was evaluated descriptively. Second, survival curves were generated for each group, and the difference in time to se- 
roconversion was compared. Finally, as the data originated from 6 separate farms with different backgrounds, the Cox proportional hazard model, calculated using the coxme package [11], was used to take into account the random effect of the farms (frailty model). The equation for the frailty model is expressed as

$$
\begin{aligned}
& \lambda(t)=\lambda_{0}(t) e^{x \beta+z b} \\
& b \sim G(0, \Sigma(\theta))
\end{aligned}
$$

where $\lambda_{0}$ denotes the baseline hazard at time $t, x$ and $z$ denote the design matrix, and $\beta$ and $b$ denote the coefficient vectors of the fixed and random effects. For this study, we assumed that $b$ is normally distributed with a zero mean and variance following vector $\Sigma$ [11]. The age of uninfected cattle as well as differences in neighboring conditions was considered as possible fixed variables. These were introduced into the model one at a time, and each model was evaluated using the likelihood ratio test to determine significance. Only variables that showed significance were incorporated into the final model.

Table 1 shows the herd inventories. A total of 245 cattle were serologically tested from the 6 farms. Out of these, 88 cattle tested positive on the initial test. The number of cattle that tested negative in at least 2 tests was 134 . Therefore, 23 cattle were excluded from analysis, primarily because of the replacement of an animal after the initial test or changes in exposure status during the observation period. Out of the 134 uninfected cattle, 81 were categorized as unexposed and 53 as exposed. From these, the number of cattle that eventually seroconverted was $3(3.7 \%)$ and 11 (20.8\%), respectively. All but one of the seroconversions were detected in the tests conducted between October and December after a previous negative test from June to August. Figure 1 shows the survival curves by group, showing that the exposed group seroconverted earlier than the unexposed

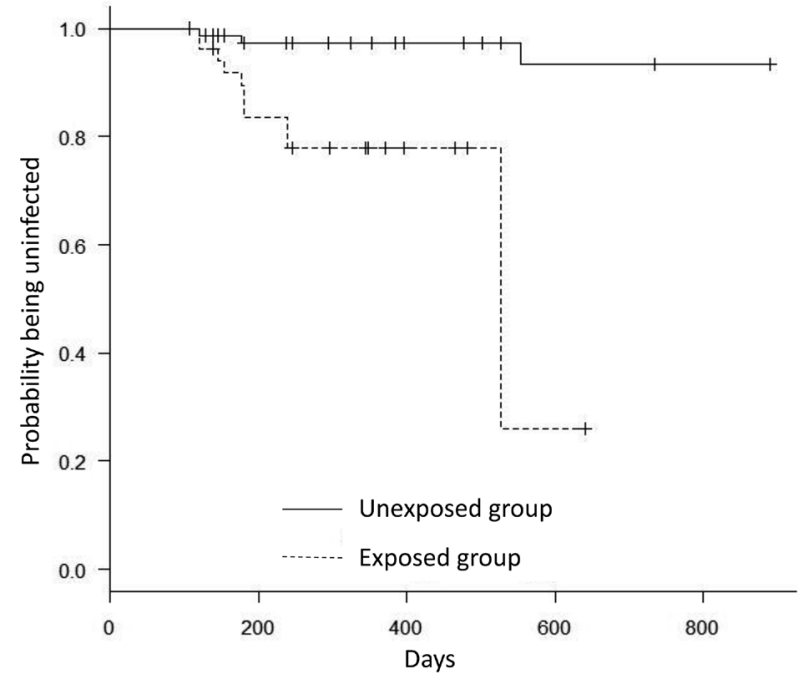

Fig. 1. Survival curves for seroconversion of uninfected cattle. Exposed group (dashed line) seroconverted earlier than the unexposed group (solid line). Censored animals are indicated as the small vertical tick-marks on each curve.

group. The exposed group showed a quick drop around days 500. This was because that, comparing to the other group, the denominator in the exposed group had decreased in the short period due to the censored (drop out) animals before days 500, and then, the impact of the seroconversion to the survival curve got larger. In the frailty model, the exposure condition was only significant variable. The hazard ratio of the exposed group compared with the unexposed group is 12.4 (95\% confidence interval: $3.0-52.0 ; P=0.001$; Table 2 ).

Results of our present analyses show that cattle adjacent to infected cattle seroconverted earlier than those without infected neighbors on BLV infected dairy farms. There are a number of possible BLV transmission routes from neighbor-

Table 1. Study cattle from six farms: herd size, actual and total number evaluated, and number of BLV seroconverters

\begin{tabular}{lccccccc}
\hline \multicolumn{1}{c}{ Farm } & 1 & 2 & 3 & 4 & 5 & 6 & Total \\
\hline Cattle serologically tested during the observation period & 43 & 43 & 56 & 26 & 26 & 51 & 245 \\
Number of serological positives among tested cattle above & 18 & 20 & 5 & 8 & 13 & 24 & 88 \\
Number of uninfected cattle tested two or more times without change & 20 & 14 & 46 & 17 & 12 & 25 & 134 \\
\hline Unexposed group (uninfected cattle without infected neighbors) & 9 & 6 & 39 & 13 & 10 & 4 & 81 \\
Number seroconverting among unexposed group & 0 & 0 & 2 & 0 & 0 & 1 & 3 \\
Exposed group (uninfected cattle having infected neighbors) & 11 & 8 & 7 & 4 & 2 & 21 & 53 \\
Number seroconverting among exposed group & 1 & 2 & 1 & 4 & 1 & 2 & 11 \\
\hline Observation period (months) & 21 & 16 & 29 & 17 & 16 & 15 & - \\
\hline
\end{tabular}

Table 2. Description of the final frailty model for the risk of seroconversion by the exposure status

\begin{tabular}{lccccc}
\hline \multicolumn{1}{c}{ Level } & Coefficient & $\begin{array}{c}\text { Standard error of } \\
\text { coefficient }\end{array}$ & $\begin{array}{c}\text { Hazard ratio } \\
(\mathrm{HR})\end{array}$ & $\begin{array}{c}95 \% \text { confidence } \\
\text { interval of HR }\end{array}$ & $P$ \\
\hline $\begin{array}{l}\text { Unexposed group } \\
\text { Exposed group }\end{array}$ & $\begin{array}{c}\text { Reference } \\
2.52\end{array}$ & 0.73 & 12.43 & $2.97,51.98$ & 0.001 \\
\hline
\end{tabular}

Number of observations $=132, \chi^{2}$ value of likelihood ratio test $=15.11(P=0.0001)$. 
ing cattle. First, tabanids (e.g., horse flies) are well-known mechanical vectors of BLV [8]. In particular, interrupted feeding of tabanids is important for efficient mechanical transmission [5]. It has been estimated that only $10 \%$ of tabanids successfully feed to repletion during the initial attempt on cattle. If feeding is interrupted, tabanids return to the same or a nearby host within seconds [6], allowing the residual blood on the mouthparts to transmit infection. This behavior also implies neighboring transmission, as infected lymphocytes would only be transmitted to adjacent animals rather than those in a different area of the barn within a limited time window for transmission. In Japan, the prevalence of blood sucking insects during the summer is one of the risk factors associated with high BLV prevalence in the dairy farms [3]. Indeed, all but one seroconversion in this study occurred at some time between summer and autumn, which largely overlapped the manifesting period of the horseflies in Japan. Therefore, the role of tabanids in the BLV transmission among neighboring cattle should be evaluated carefully in future.

On the other hand, another route of BLV transmission without the aid of tananids has also been confirmed experimentally in insect-proof pens [4]. This also likely occurred during this study, because of one seroconversion in the exposed group occurred during non-summer seasons. Considering that infected and uninfected cattle remained in close proximity in tie-stalls regardless of the season, one possibility would be the transmission of infected lymphocytes via milking practice in spite of its unknown mechanism. However, it is at least known that milk and colostrum of infected cattle include infected lymphocytes [1]. In case that infected animal is milked prior to the uninfected one, teats of the uninfected might have a chance to get exposed to contaminated milk. Further experiments, such as tracking milking machine use and following seroconversion, would be necessary to determine the role this may play in transmission.

While this study provides an important starting point for examining local transmission risk in dairy farms, it has several limitations. As samples were collected at 4 to 6 month intervals, the actual time for seroconversion might be shorter than our observations. Increased sampling frequency would allow for more precise determination of seroconversion time, which may provide more information on possible transmission routes. As no test has perfect sensitivity and specificity, classification of cattle into the exposed and unexposed groups as well as the consecutive test results obtained by ELISA testing during the observation period may not be completely accurate. However, these errors should be equally distributed between both groups and should not significantly impact intergroup comparisons. Therefore, the prolonged time to seroconversion is unlikely to be an artefact of testing and represents a true difference between groups. In addition, we have not evaluated the effect of proviral load of the infected cattle, which might highly influence the BLV transmission. This would be a crucial point for the better understanding of the mechanisms of our observational results.

In conclusion, this study shows a significant transmission risk of BLV to uninfected cattle from neighboring infected cattle in the tie-stall houses. This finding provides support for animal health authorities to segregate infected cattle to reduce BLV transmission, ultimately decreasing prevalence.

ACKNOWLEDGMENTS. This study was conducted under the approval of the Ministry of Agriculture, Forestry and Fisheries as a part of the official animal health research project on BLV control from 2010 to 2012. We appreciate the Ministry's financial support. We are also grateful to Dr. Shuji Yoneyama, Dr. Keisuke Hamatani, Dr. Ryo Ikegami and Dr. Yoji Ishikura from Tochigi, Toyama and Shimane prefectures, respectively, for their generous time and efforts in coordinating farmers and collecting data, as well as the six farmers who kindly provided their cattle health information.

\section{REFERENCES}

1. Ferrer, J. F. and Piper, C. E. 1981. Role of colostrum and milk in the natural transmission of the bovine leukemia virus. Cancer Res. 41: 4906-4909. [Medline]

2. Kettmann, R., Burny, A., Callebaut, I., Droogmans, L., Mammerickx, M., Willems, L. and Portetelle, D. 1994. Bovine leukemia virus. pp. 39-81. In: The Retroviridae. New York Plenum Press, New York.

3. Kobayashi, S., Hidano, A., Tsutsui, T., Yamamoto, T., Hayama, Y., Nishida, T., Muroga, N., Konishi, M., Kameyama, K. and Murakami, K. 2014. Analysis of risk factors associated with bovine leukemia virus seropositivity within dairy and beef breeding farms in Japan: a nationwide survey. Res. Vet. Sci. 96: 47-53. [Medline] [CrossRef]

4. Kono, Y., Sentsui, H., Arai, K., Ishida, H. and Irishio, W. 1983. Contact transmission of bovine leukemia virus under insect-free conditions. Japanese. J. Vet. Sci. 45: 79-82.

5. Magnarelli, L. A. and Anderson, J. F. 1980. Feeding behavior of Tabanidae (Diptera) on cattle and serological analysis of partial blood meals. Environ. Entomol. 9: 664-667. [CrossRef]

6. Mullens, B. A. 2002. Horse flies and deer flies (Tabanidae). pp. 263-277 In: Medical and Veterinary Entomology. San Diego Academic Press, San Diego.

7. Murakami, K., Kobayashi, S., Konishi, M., Kameyama, K. and Tsutsui, T. 2013. Nationwide survey of bovine leukemia virus infection among dairy and beef breeding cattle in Japan from 2009-2011. J. Vet. Med. Sci. 75: 1123-1126. [Medline] [CrossRef]

8. Ohshima, K., Okada, K., Numakunai, S., Yoneyama, Y., Sato, S. and Takahashi, K. 1981. Evidence on horizontal transmission of bovine leukemia virus due to blood-sucking tabanid flies. Jpn. J. Vet. Sci. 43: 79-81. [Medline] [CrossRef]

9. Rodríguez, S. M., Florins, A., Gillet, N., de Brogniez, A., Sánchez-Alcaraz, M. T., Boxus, M., Boulanger, F., Gutiérrez, G., Trono, K., Alvarez, I., Vagnoni, L. and Willems, I. 2011. Preventive and therapeutic strategies for bovine leukemia virus: lessons for HTLV. Viruses 3: 1210-1248. [Medline] [CrossRef]

10 R Development Core Team 2011. R: A language and environment for statistical computing. R Foundation for Statistical Computing Vienna Austria. Cited 2014 May 25. http:/www.Rproject.org/

11. Therneau, T. Mixed Effects Cox Models. 2012. Cited 2014 Apr. 14. http://cran.r-project.org/web/packages/coxme/coxme.pdf 